\title{
Impact of dronedarone in atrial fibrillation and flutter on stroke reduction
}

\author{
This article was published in the following Dove Press journal: \\ Clinical Interventions in Aging \\ 25 March 2010 \\ Number of times this article has been viewed
}

\author{
Christine Benn Christiansen' \\ Christian Torp-Pedersen' \\ Lars Køber ${ }^{2}$ \\ 'Department of Cardiology, Gentofte \\ Hospital, University of Copenhagen, \\ Hellerup, Denmark; ' ${ }^{2}$ epartment of \\ Cardiology, Rigshospitalet, University \\ of Copenhagen, Copenhagen, \\ Denmark
}

\begin{abstract}
Background: Dronedarone has been developed for treatment of atrial fibrillation (AF) or atrial flutter (AFL). It is an amiodarone analogue but noniodinized and without the same adverse effects as amiodarone.
\end{abstract}

Objective and methods: This is a review of 7 studies (DAFNE, ADONIS, EURIDIS, ATHENA, ANDROMEDA, ERATO and DIONYSOS) on dronedarone focusing on efficacy, safety and prevention of stroke. There was a dose-finding study (DAFNE), 3 studies focusing on maintenance of sinus rhythm (ADONIS, EURIDIS and DIONYSOS), 1 study focusing on rate control (ERATO) and 2 studies investigating mortality and morbidity (ANDROMEDA and ATHENA).

Results: The target dose for dronedarone was established in the DAFNE study to be $400 \mathrm{mg}$ twice daily. Both EURIDIS and ADONIS studies demonstrated that dronedarone was superior to placebo for maintaining sinus rhythm. However, DIONYSOS found that dronedarone is less efficient at maintaining sinus rhythm than amiodarone. ERATO concluded that dronedarone reduces ventricular rate in patients with chronic AF. The ANDROMEDA study in patients with severe heart failure was discontinued because of increased mortality in dronedarone group. Dronedarone reduced cardiovascular hospitalizations and mortality in patients with AF or AFL in the ATHENA trial. Secondly, according to a post hoc analysis a significant reduction in stroke was observed (annual rate $1.2 \%$ on dronedarone vs $1.8 \%$ on placebo, respectively [hazard ratio 0.66 , confidence interval 0.46 to $0.96, P=0.027]$ ). In total, 54 cases of stroke occurred in 3439 patients (crude rate $1.6 \%$ ) receiving dronedarone compared to 76 strokes in 3048 patients on placebo (crude rate $2.5 \%$ ), respectively.

Conclusion: Dronedarone can be used for maintenance of sinus rhythm and can reduce stroke in patients with $\mathrm{AF}$ who receive usual care, which includes antithrombotic therapy and heart rate control.

Keywords: atrial fibrillation, stroke, dronedarone

\section{The risk of stroke in patients with atrial fibrillation}

Stroke associated with atrial fibrillation (AF) is often disabling and life threatening. Stroke is the second most common cause of death after ischemic heart disease in the world with 4.38 million deaths in developed countries and almost 3 million in developing countries. ${ }^{1}$ Stroke causes $10 \%$ to $12 \%$ of deaths in industrialized countries. Almost $90 \%$ of deaths caused by stroke occur in people over 65 years of age. ${ }^{2}$ The Framingham study showed that AF is an independent risk factor for development of stroke, particularly in the elderly. ${ }^{3}$ Given that AF is a known risk factor for stroke, it seems appealing that a drug with antiarrhythmic effect can reduce the incidence of stroke in patients with AF.
Correspondence: Lars Køber

Department of Cardiology, Rigshospitalet, University of Copenhagen, Blegdamsvej 9, 2100 Copenhagen, Denmark

$\mathrm{Tel}+4535453376$

Fax +45 35452341

Email lk@heart.dk 
However, previous studies point in the opposite direction: Rhythm Control versus Rate Control for Atrial Fibrillation and Heart Failure, Atrial Fibrillation Follow-up Investigation of Rhythm Management (AFFIRM) showed that antiarrhythmic drugs reduced the recurrence of AF, but did not show any reduction in stroke. ${ }^{4}$ In a meta-analysis of randomized trials of rate vs rhythm control for AF from 2005 there was an insignificant increase in stroke in the group of patients who received rhythm control. ${ }^{5}$ The incidence of stroke has declined due to treatment with oral anticoagulant therapy, and is around $1.4 \%$ /year in patients with $\mathrm{AF}^{6,7}$ However, $\mathrm{AF}$ is often associated with advanced age and concomitant disease that disposes to stroke: hypertension, hypercholesterolemia, diabetes and heart failure. Prevention of stroke may require treatment of several conditions in order to become effective. Thus, it may not be surprising that antiarrhythmic therapy have not been able to prevent stroke in randomized clinical trials.

\section{Dronedarone, a new antiarrhythmic drug}

Dronedarone has been developed by Sanofi-Aventis for treatment of AF or atrial flutter (AFL). Dronedarone is a benzofuran derivative and primarily a class III antiarrhythmic drug but with properties from all four classes of the Vaughan-Williams classification. It is an amiodarone analogue but noniodinized and without many of the adverse effects associated with amiodarone. It has antiadrenergic properties and inhibits multiple transmembrane potassium currents, including the delayed rectifier current (both the rapid and slow components (IKr and IKs, respectively), the ultrarapid rectifier current (Ikur), the inward rectifier current (IK1), and the transient outward current (ITo), as well as sodium and L-type calcium currents. ${ }^{89}$ Dronedarone is approximately 100 times more potent than amiodarone and sotalol on the acetylcholine receptor-operated $\mathrm{K}^{+}$current which is important when vagal tone plays a role in the genesis of $\mathrm{AF}{ }^{10}$ The removal of iodine is thought to be the reason for the lack of typical amiodarone side effects such as discoloration of skin, and affects on of lung, liver, and thyroid gland. Dronedarone is less lipophilic and has a much shorter half-life than amiodarone because of a methylsulfonamide group. Dronedarone interferes with the metabolism of digoxin, but not with the metabolism of warfarin. ${ }^{11}$ It is hepatically metabolized and excreted with feces.

\section{Clinical effects of dronedarone}

There are 7 important studies with dronedarone, of which two were powered to show effects on mortality and morbidity.
The DAFNE trial (dronedarone Atrial Fibrillation study after Electrical Cardioversion) studied the most appropriate dose of dronedarone to maintain sinus rhythm after cardioversion. ${ }^{12}$ The study included 199 patients with persistent AF. Patients with severe heart failure or left ventricular ejection fraction $(\mathrm{LVEF})<35 \%$ were excluded. The patients were randomized to 4 different groups and received either placebo or dronedarone in doses of $400 \mathrm{mg}, 600 \mathrm{mg}$ or $800 \mathrm{mg}$ twice daily. DAFNE showed that the most appropriate dose of dronedarone was $400 \mathrm{mg}$ twice daily for prevention of AF relapses in patients after cardioversion. It was somewhat puzzling that higher doses of dronedarone were less efficient than $400 \mathrm{mg}$ twice daily. Multivariable adjustment for baseline characteristics resulted in identical results. The most likely explanation is that higher dosages were limited by adverse events resulting in discontinuation of the drug. There was no report of stroke in any of the dronedarone groups or in the placebo group. There was one case of thrombosis $(1.3 \%)$ in the group that received $800 \mathrm{mg}$ twice daily and none in the other groups.

EURIDIS (European Trial in AF or AFL Patients receiving dronedarone for the Maintenance of Sinus Rhythm) and ADONIS (American-Australian-African Trial with dronedarone in AF or AFL Patients for the Maintenance of Sinus Rhythm) were two identical studies of which one was European and one non-European. ${ }^{13}$ All included patients had a history of AF but did not have AF at the time of enrolment. The purpose of the studies was to prevent recurrence of AF with dronedarone $(n=828)$ compared to placebo $(n=409)$. In both EURIDIS and ADONIS dronedarone increased the time to recurrence of AF significantly compared to the placebo: 96 days in 41 days $(P$ value $=0.01)$ and 158 days vs 59 days $(P=0.002)$, respectively. In the European trial $67 \%$ of the patients had had a recurrence of AF vs $77.5 \%$ in the placebo group (hazard ratio [HR] 0.78, 95\% confidence interval [CI] 0.64 to $0.96, P=0.01)$. In the non-European trial $61.1 \%$ of patients in the dronedarone group had had a recurrence of $\mathrm{AF}$ vs $72.8 \%$ in the placebo group (HR 0.73 , $95 \%$ CI 0.59 to $0.89, P=0.002$ ). These high rates of recurrence of $\mathrm{AF}$ in EURIDIS and ADONIS could lead to the assumption that dronedarone is less efficient in maintaining sinus rhythm than other antiarrhythmic drugs. However, the populations of different studies vary considerably, which makes direct comparison impossible.

There was no significant difference in the number of strokes reported as there were 4 strokes $(0.5 \%)$ in the dronedarone group and 3 strokes $(0.7 \%)$ in the placebo group $(P=0.69)$. Stroke included cerebral-artery embolism, 
cerebrovascular accident, cerebral infarction, and transient ischemic attack.

The efficacy and safety of dronedarone for the control of ventricular rate during AF (ERATO) study assessed the efficacy of dronedarone in the control of ventricular rate in patients with permanent AF. ${ }^{14}$ The study enrolled 85 patients in the dronedarone group receiving $400 \mathrm{mg}$ dronedarone twice daily and 89 in the placebo group. All patients had a history of symptomatic, permanent AF for minimum 6 months and resting ventricular rate of at least 80 beats per minute. ERATO demonstrated a highly significant rate reduction in patients with permanent AF. At day 14 the mean ventricular rate was measured to be 11.7 beats less in the dronedarone group than at day $0(P<0.0001)$. During submaximal and maximal exercise the mean ventricular rate at day 14 was reduced by 25.6 and 27.4 beats, respectively, vs a 2.2 and 2.9 beats per minute reduction in the placebo group (both $P<0.0001$ ). There is no report of stroke in ERATO. The serious cardiovascular events included 3 myocardial infarctions ( 1 in dronedarone vs 2 in placebo group) and 1 heart failure (in the dronedarone group) and 1 unstable angina (in the placebo group). ${ }^{14}$

A comparison of dronedarone and amiodarone was made in the efficacy and safety of dronedarone vs amiodarone for the maintenance of sinus rhythm in patients with AF (DIONYSOS) trial. The 504 patients scheduled for cardioversion for AF or AFL were randomized to amiodarone or dronedarone (400 $\mathrm{mg}$ twice daily), and the primary endpoint was the combined endpoint of recurrence of $\mathrm{AF}$ or premature discontinuation. DIONYSOS has not been published, but a press release (http://en.sanofi-aventis.com/ binaries/20081223_dionysos_fe_en_en_tcm28-23624.pdf) reveals that dronedarone has a higher rate of recurrence of AF or premature discontinuation after a year than amiodarone (HR approximately $1.6 ; P<0.01$ ). Recurrence of AF occurred for 158 patients in the dronedarone group vs 107 in the placebo group. The significant reduction in recurrence of AF by amiodarone is consistent with an indirect metaanalysis based on 4 studies of amiodarone and 4 studies of dronedarone. ${ }^{15}$

\section{Is dronedarone safe?}

The antiarrhythmic trial with dronedarone in moderate to severe congestive heart failure evaluating morbidity decrease (ANDROMEDA) trial was a placebo-controlled multicenter study performed in patients admitted to hospital with moderate to severe heart failure and reduced left ventricular ejection fraction (LVEF). ${ }^{16}$ The study was performed in order to show a reduction in morbidity and mortality in patients with severe heart failure. The study was planned to include 1000 patients and last for a minimum of 2 years. Every patient was treated for a minimum of 12 months. The primary end point was death from any cause or hospitalization for worsening heart failure. Secondary end points included all cause mortality and hospitalization for cardiovascular causes. After inclusion of 627 patients (310 in the dronedarone group and 317 in the placebo group), the study was discontinued, when the Data, Safety and Management Board (DSMB) found that dronedarone was associated with a significantly higher mortality. After a median 2-month follow-up, 25 patients in the dronedarone group and 12 patients in the control group had died, respectively. DSMB recommended that the study was stopped prematurely. Based on the small number of events it is difficult to know if this decision was correct. However, the increased mortality in the dronedarone was discovered at the first look at data, and repeated 1 month later. The steering committee discussed whether the advice of the DSMB should be followed, and decided to stop the study. Subgroup analyses could not identify a specific group of patients to which the increased risk was confined, but the risk seemed to be associated with the sickest patients. In the dronedarone group an increased number had an increase in serum creatinine. It was suggested that dronedarone might decrease renal function but this suggestion was later rejected. On the contrary, a study among 12 healthy males showed that dronedarone increases serum creatinine (increasing tubular secretion) without decreasing renal function. ${ }^{17}$ In ANDROMEDA, $4(5.6 \%)$ in the dronedarone group and $3(6.0 \%)$ in the placebo group suffered from stroke requiring a first hospitalization.

As the population in ANDROMEDA consisted of patients with moderate to severe heart failure, a large study in patients of medium risk was planned following the results of ANDROMEDA. The study was done in patients with current or recent $\mathrm{AF}$ in order to show if dronedarone could affect mortality or morbidity. A placebo-controlled, double-blind, parallel-arm trial to assess the efficacy of dronedarone $400 \mathrm{mg}$ twice daily for the prevention of cardiovascular hospitalization or death from any cause in patients with AF/AFL (ATHENA) was a placebo controlled multicenter study including 4628 patients. The primary end point was death or hospitalization for a cardiovascular reason. Inclusion criteria were a history of paroxysmal or persistent $\mathrm{AF}$ and at least one of following risk factors: age of minimum 70 years, arterial hypertension (with ongoing therapy involving at least two antihypertensive drugs of different classes), diabetes mellitus, previous stroke, 
transient ischemic attack, systemic embolism, minimum left atrial diameter of $50 \mathrm{~mm}$ or LVEF of $40 \%$ or less. At the time of enrollment 1176 (25\%) of the patients had either AF or AFL. A history of NYHA class II or III heart failure was present in 979 patients. However, only 179 patients (4\%) had LVEF $<35 \%$. The overall mortality figures were lower than expected, so the steering committee changed the inclusion criteria to enrich the risk profile of the overall study population. With the revised criteria patients aged 75 years or older could be included whether or not they had any of the specified risk factors. Patients aged 70 years or older could be included if they had at least one of the risk factors. Patients younger than 70 years could no longer be included. Patients included in the study had an intermediate to high risk for stroke and other cardiovascular events. In total, $60 \%$ received oral anticoagulant therapy (OAC) and one-fourth of these received both OAC and an antiplatelet agent. Mean followup period was 21 months. Exclusion criteria included permanent AF, severe heart failure (NYHA class IV), unstable hemodynamic condition, bradycardia, planned major surgery and glomerular filtration rate of less than $10 \mathrm{~mL}$ per minute. The population in ATHENA therefore varied considerably from the population in ANDROMEDA as the ATHENA patients were all clinically stable and had non-permanent AF. In contrast, patients in the ANDROMEDA study were hospitalized and the majority did not have AF. Thus, in the ATHENA study investigators excluded patients similar to those participating in ANDROMEDA, and included only patients for whom dronedarone was developed. In ATHENA, 734 patients $(31.9 \%)$ in the dronedarone group reached the primary end point of either death from any cause or hospitalization for cardiovascular reasons vs 917 (39.4\%) in the placebo group (HR 0.76; 95\% CI 0.69 to 0.84 ; $P<0.001$ ), respectively. Death from any cause did not differ significantly between the two groups, as $116(5 \%)$ in the dronedarone group vs $139(6.0 \%)$ in the placebo group died, respectively (HR $0.84,95 \%$ CI, 0.66 to $1.08 ; P=0.18$ ). Hospitalization for cardiovascular reasons occurred in 675 (29.3\%) in the dronedarone group vs 859 (36.9\%) in the placebo group, respectively (HR $0.74 ; 95 \% \mathrm{CI} 0.67$ to $0.82 ; P<0.001$ ). This difference in hospitalizations between the two groups was mainly caused by a reduction in the number of hospitalizations for AF. However, hospitalizations for AF were often related to other cardiovascular events (ie, incompensation, stroke or myocardial infarction). Secondary endpoints included death from cardiovascular causes and any hospitalization due to cardiovascular events. Death from cardiovascular reasons occurred in $63(2.7 \%)$ in the dronedarone group vs
$90(3.9 \%)$ in the placebo group (HR $=0.71 ; 95 \%$ CI 0.51 to $0.98 ; P=0.03)$, respectively. Also, there were 26 deaths from cardiac arrhythmia (1.1\% of patients) in the dronedarone group and $48(2.1 \%)$ in the placebo group (HR, 0.55; $95 \%$ CI, 0.34 to $0.88, P=0.01$ ), respectively. Any hospitalization due to any cardiovascular event or death from any cause occurred in $1253(54.5 \%)$ cases in the dronedarone group vs $1668(71.7 \%$ ) in the placebo group (HR 0.76, 95\% (CI 0.68 to 0.84$), P<0.001$ ). Overall, the ATHENA study demonstrated that in elderly patients with nonpermanent AF and additional risk factors, dronedarone was safe and reduced cardiovascular morbidity/mortality.

\section{How does dronedarone influence the risk of stroke?}

The positive outcome in the ATHENA trial was mainly driven by a reduction in cardiovascular hospitalizations. From the published data it is difficult to see how many of these events were related to an effect on $\mathrm{AF}$, and to see if dronedarone has additional effects not previously anticipated.

In a post hoc analysis deaths were categorized into four subgroups: cardiac, arrhythmic; cardiac, nonarrhythmic; vascular, noncardiac; and nonvascular. Information was then gathered from hospital reports, death reports and adverse event reports. All incidents of preferred terms for adverse effects that contained the word "stroke", "cerebrovascular accident" or "cerebellar hemorrhage" were counted as stroke. Strokes that did not lead to hospitalizations or death were reported as adverse effects. The total number of strokes was 46 (annual event rate of 1.2\%) in the dronedarone group and 70 (annual event rate of 1.8\%) in the placebo group, respectively (HR 0.66 , CI 0.46 to $0.96, P=0.027$ ). Figure 1 shows the cumulative risk of stroke and the composite outcome of stroke, acute coronary syndrome or cardiovascular death. Ischemic strokes accounted for 33 (annual event rate $0.9 \%$ ) in the dronedarone group and 49 (annual event rate $1.3 \%$ ) in the placebo group, respectively (HR 0.68, CI 0.44 to $1.05, P=0.08)$. Hemorrhagic strokes were 6 in both groups (annual event rate $0.2 ; P=0.99$ ). Subgroup analysis to investigate what baseline characteristics were predictive of a response to dronedarone in response to stroke showed that patients with a $\mathrm{CHADS}_{2}$ score $\geq 2$ had a significantly greater effect of dronedarone than patients with a $\mathrm{CHADS}_{2}$ score of $\leq 1 .{ }^{18}$

In EURIDIS and ADONIS the overall incidence of stroke was $0.3 \%(\mathrm{n}=4)$ in the dronedarone vs $0.5 \%$ $(\mathrm{n}=3)$ in the placebo group. ERATO and DAFNE, which were short-term studies, did not report any cases 
of stroke, and data from DIONYSOS are not available. In the ANDROMEDA trial stroke occurred in $4(1.3 \%)$ in the dronedarone group and $3(0.9 \%)$ in the placebo group, respectively. In total, 54 cases of stroke occurred in 3439 patients (crude rate 1.6\%) receiving dronedarone compared to 76 strokes in 3048 patients on placebo (crude rate $2.5 \%$ ), respectively. The information on the overall risk of stroke associated with dronedarone originates mainly from the ATHENA trial.
The reduction in stroke demonstrated in the ATHENA trial may have important clinical implications. This effect has never been demonstrated with any other antiarrhythmic drug, but the mechanism is uncertain. Suppression of AF is the most obvious explanation, but the details of the information collected during the ATHENA trial do not allow us to conclude this. Secondly, patients with AF that persisted during the entire study also had a reduction (nonsignificant) in stroke. Dronedarone had at least two other effects that could have

A

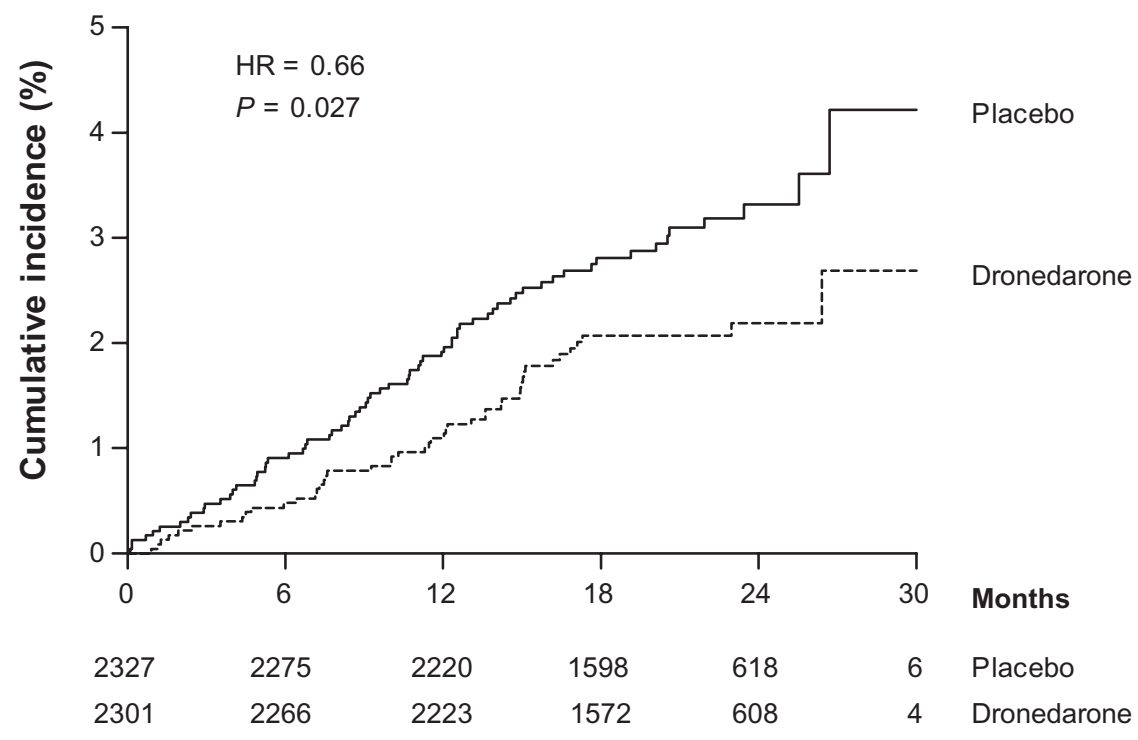

B

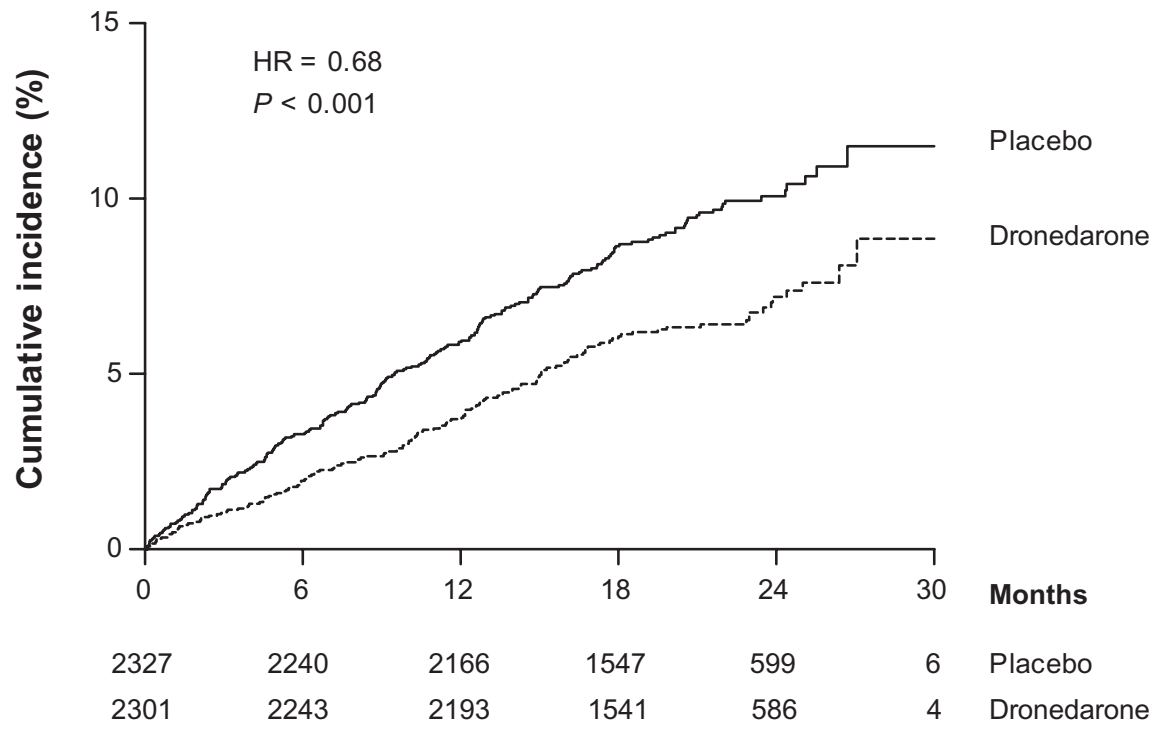

Figure I The cumulative risk of stroke (A) and the composite outcome of stroke, acute coronary syndrome or cardiovascular death (B). Reproduced with permission from Connolly SJ, Crijns HJ, Torp-Pedersen C, et al. Analysis of stroke in ATHENA: a placebo-controlled, double-blind, parallel-arm trial to assess the efficacy of dronedarone 400 mg BID for the prevention of cardiovascular hospitalization or death from any cause in patients with atrial fibrillation/atrial flutter. Circulation. 2009;। 20:I I74-II80.18 Copyright $($ ) 2009 Wolters Kluwer Health. 
resulted in a reduction in stroke. Heart rate is reduced with dronedarone and there was a significant reduction in blood pressure in the dronedarone group (approximately $3 \mathrm{mmHg}$ in both systolic and diastolic blood pressure) compared to the placebo group. These changes in blood pressure are modest, but may have importance in a group of patients with many risk factors for stroke. The most important limitation of the findings of the post hoc analysis of ATHENA is that the reduction in stroke was not anticipated and therefore not prespecified, but was found retrospectively. The results of the post hoc analysis do not provide any information on why antiarrhythmic drugs might be able to reduce the risk of stroke, but they do provide reasons for future trials to investigate if similar results can be repeated and what mechanisms lie behind. Until now the reasons for pursuing rhythm control in AF have been to improve quality of life and reduce symptoms. However, if stoke can be prevented this will open a new scenario for treatment of patients at higher risk of stroke. In this context it is important to note that the effect on stroke was only seen in patients with a $\mathrm{CHADS}_{2}$ score $\geq 2(P=0.03)$.

\section{Which patients should be treated with dronedarone?}

The FDA approval of dronedarone suggests that it may be used in patients with paroxysmal or persistent AF or AFL who are in sinus rhythm or will be cardioverted. Patients should have additional risk factors defined as age above 70 years, diabetes, hypertension, prior stroke, increased size of the left atrium or depressed LVEF $(<40 \%)$. These were the criteria for the ATHENA population. Contraindications to dronedarone are NYHA class IV, or class II or III with a recent episode of hospitalization, ie, the ANDROMEDA population. This reflects the available data, but physicians may speculate if patients for whom rate control is a target could be candidates for dronedarone treatment. The available studies have mainly focused on rhythm control, except for the ERATO study that showed a significant reduction in heart rate with dronedarone. Whether the beneficial effect of dronedarone found in the ATHENA trial can be extrapolated to patients with permanent $\mathrm{AF}$ is unknown. Thus, dronedarone should primarily be used for rhythm control. However, if patients develop permanent AF on dronedarone this does not imply that treatment necessarily has to be discontinued. The observed reduction in rate may be beneficial to the patients.

\section{Conclusion}

Dronedarone is developed as an antiarrhythmic drug and the EURIDIS/ADONIS studies found that dronedarone can decrease the recurrence of AF. In the ATHENA trial it was demonstrated that dronedarone may reduce cardiovascular mortality and morbidity. Importantly, in a post hoc analysis stroke was observed to be significantly reduced in elderly patients with AF. Since AF is a known risk factor for stroke, this result may not be surprising, but previous studies have not found any decreased risk in patients treated with antiarrhythmic drugs compared to patients treated with rate-controlling drugs. Thus, dronedarone may be useful for treatment of elderly patients with non-permanent AF.

\section{Acknowledgments/disclosures}

Christian Torp-Pedersen served as member of the steering committee on both ATHENA and ANDROMEDA and has received consultancy fees from Sanofi-Aventis.

Lars Køber was principal investigator for the ANDROMEDA trial and has received consultancy fees from SanofiAventis.

\section{References}

1. Murray CJ, Lopez AD. Mortality by cause for eight regions of the world: Global Burden of Disease Study. Lancet. 1997;349:1269-1276.

2. Bonita R. Epidemiology of stroke. Lancet. 1992;339:342-344.

3. Wolf PA, Abbott RD, Kannel WB. Atrial fibrillation as an independent risk factor for stroke: the Framingham Study. Stroke. 1991;22:983-988.

4. Sherman DG, Kim SG, Boop BS, et al. Occurrence and characteristics of stroke events in the Atrial Fibrillation Follow-up Investigation of Sinus Rhythm Management (AFFIRM) study. Arch Intern Med. 2005;165:1185-1191

5. de Denus S, Sanoski CA, Carlsson J, Opolski G, Spinler SA. Rate vs rhythm control in patients with atrial fibrillation: a meta-analysis. Arch Intern Med. 2005;165:258-262.

6. Stroke Prevention in Atrial Fibrillation Study. Final results. Circulation. 1991;84:527-539

7. The effect of low-dose warfarin on the risk of stroke in patients with nonrheumatic atrial fibrillation. The Boston Area Anticoagulation Trial for Atrial Fibrillation Investigators. N Engl J Med. 1990;323:1505-1511.

8. Gautier P, Guillemare E, Marion A, Bertrand JP, Tourneur Y, Nisato D. Electrophysiologic characterization of dronedarone in guinea pig ventricular cells. J Cardiovasc Pharmacol. 2003;41:191-202.

9. Chatelain P, Meysmans L, Matteazzi JR, Beaufort P, Clinet M. Interaction of the antiarrhythmic agents SR 33589 and amiodarone with the beta-adrenoceptor and adenylate cyclase in rat heart. Br J Pharmacol. 1995;116:1949-1956.

10. Guillemare E, Marion A, Nisato D, Gautier P. Inhibitory effects of dronedarone on muscarinic $\mathrm{K}^{+}$current in guinea pig atrial cells. J Cardiovasc Pharmacol. 2000;36:802-805.

11. Zimetbaum PJ. Dronedarone for atrial fibrillation - an odyssey. $N$ Engl J Med. 2009;360:1811-1813.

12. Touboul P, Brugada J, Capucci A, Crijns HJ, Edvardsson N, Hohnloser SH. Dronedarone for prevention of atrial fibrillation: a doseranging study. Eur Heart J. 2003;24:1481-1487.

13. Singh BN, Connolly SJ, Crijns HJ, et al. Dronedarone for maintenance of sinus rhythm in atrial fibrillation or flutter. $N$ Engl $J$ Med. 2007;357:987-999.

14. Davy JM, Herold M, Hoglund C, et al. Dronedarone for the control of ventricular rate in permanent atrial fibrillation: the Efficacy and safety of dRonedArone for the cOntrol of ventricular rate during atrial fibrillation (ERATO) study. Am Heart J. 2008;156:527:e521-e529. 
15. Piccini JP, Hasselblad V, Peterson ED, Washam JB, Califf RM, Kong DF. Comparative efficacy of dronedarone and amiodarone for the maintenance of sinus rhythm in patients with atrial fibrillation. J Am Coll Cardiol. 2009;54:1089-1095.

16. Kober L, Torp-Pedersen C, McMurray JJ, et al. Increased mortality after dronedarone therapy for severe heart failure. $N$ Engl $J$ Med. 2008;358:2678-2687.

17. Tschuppert $Y$, Buclin T, Rothuizen LE, et al. Effect of dronedarone on renal function in healthy subjects. Br J Clin Pharmacol. 2007;64: 785-791.
18. Connolly SJ, Crijns HJ, Torp-Pedersen C, et al. Analysis of stroke in ATHENA: a placebo-controlled, double-blind, parallel-arm trial to assess the efficacy of dronedarone $400 \mathrm{mg}$ BID for the prevention of cardiovascular hospitalization or death from any cause in patients with atrial fibrillation/atrial flutter. Circulation. 2009;120:1174-1180.
Clinical Interventions in Aging

\section{Publish your work in this journal}

Clinical Interventions in Aging is an international, peer-reviewed journal focusing on evidence-based reports on the value or lack thereof of treatments intended to prevent or delay the onset of maladaptive correlates of aging in human beings. This journal is indexed on PubMed Central, MedLine, the American Chemical Society's 'Chemical

\section{Dovepress}

Abstracts Service' (CAS), Scopus and the Elsevier Bibliographic databases. The manuscript management system is completely online and includes a very quick and fair peer-review system, which is all easy to use. Visit http://www.dovepress.com/testimonials.php to read real quotes from published authors.

Submit your manuscript here: http://www.dovepress.com/clinical-interventions-in-aging-journal 